\title{
Prevalence of Hypodontia in the Permanent Dentition of Macedonian Population
}

\section{SUMMARY}

Hypodontia or tooth agenesis is a condition at which the patient is missing one or more teeth due to a failure of those teeth to develop. This is not only an aesthetic, but also a functional deficiency. The incidence of congenitally missing teeth depends on etiological factors that affect tooth development, as well as which dentition is concerned, sex or race and geographic distribution. The tooth agenesis is mostly seen in teeth that are formed last in a given class (lateral incisors, second premolars and third molars). The aim of this study was to calculate the prevalence of congenitally missing teeth in population of FYROM and, through a review of the literature, to compare these results to other populations in the world.

For this purpose a retrospective, transversal and cross-sectional study was made, where dental history and anamnesis of 8160 patients (3671 males and 4489 females) were examined, as well as their panoramic radiographs. The patients were 8-18 years old. The data was statistically analyzed with programme Statistica 7.0. The prevalence of hypodontia population of FYROM was $7.52 \%$. Most commonly congenitally missing tooth in patients with hypodontia was mandibular second premolar (35.5\% left and $34.53 \%$ right). Tooth agenesis predominated in females and in ethnic Albanian population, without significance.

Keywords: Hypodontia, prevalence; Tooth Agenesis; FYROM
Darko Pop Acev ${ }^{1}$, Julijana Gjorgova ${ }^{2}$

${ }^{1}$ Private Dental Office "Pop Acevi" Skopje, FYROM

${ }^{2}$ Ss. Cyril and Methodius University in Skopje Department of Orthodontics, Skopje, FYROM

\section{ORIGINAL PAPER (OP)}

Balk J Dent Med, 2014; 18:93-98

\section{Introduction}

Hypodontia or tooth agenesis is a condition at which the patient is missing one or more teeth due to a failure of those teeth to develop. This is due to an absence of their germ. Hypodontia describes a situation where the patient is missing up to 6 teeth, excluding the third molars. If a patient is missing more than 6 teeth, excluding the third molars, that is oligodontia, whilst the condition where all teeth are missing is called anodontia.

Tooth agenesis is the most common congenital dental anomaly ${ }^{1-3}$. It is not only an aesthetic, but also a functional deficiency ${ }^{4-6}$. The patients with tooth agenesis in permanent dentition may have masticatory dysfunction as well as irregular pronunciation. Additionally, tooth agenesis can be followed by other conditions, as delayed tooth eruption, abnormalities in tooth shape, captured primary teeth, ectopic canines or taurodontism ${ }^{7-9}$. Also, tooth agenesis of permanent teeth has negative effect on the sagittal development of the jaw, as well as an increased vertical overlap (overbite) ${ }^{10}$.

Lack of front teeth or agenesis of more than 2 teeth in the same quadrant is an indication for orthodontic treatment ${ }^{11-12}$. Thus, for the clinicians, this presents a common problem in dental practice and its treatment is a real challenge. Therefore, examination of the prevalence of tooth agenesis has a big role in both, early diagnosis and the plan of the treatment ${ }^{5}$. This will prevent complications of hypodontia, such as malocclusions and insufficient development of alveolar bone ${ }^{11,13,14}$.

Many etiological studies have been made worldwide to define the prevalence of this anomaly for a certain country, city or a region. Usually they are retrospective radiographic studies. Reviewing the literature we can assume that presence of tooth agenesis is different for different populations. The prevalence of hypodontia (excluding the third molars) in the permanent dentition among different populations is from $2.6 \%-11.3 \%{ }^{15}$ (Tab. 1). 
Table 1. Prevalence of hypodontia among different population

\begin{tabular}{llllccccc}
\hline Author & Year & Country & Sample type & Sample Size & Female (\%) & Male (\%) & M:F & Prevalence (\%) \\
\hline Nik-Hussein NN & 1989 & Malaysia & SC & 1583 & 3.5 & 2.2 & 0.629 & 2.8 \\
Aasheim et al. & 1993 & Norway & SC & 1953 & 7.2 & 5.8 & 0.806 & 6.5 \\
Sterzik et al. & 1994 & Germany & OP & 3238 & - & - & - & 8.1 \\
Ng'ang'a RN, Ng'ang'a PM & 2001 & Kenya & OP & 615 & 5.3 & 7.2 & 1.358 & 6.3 \\
Nordgarden et al. & 2002 & Norway & PuDP & 430 & 5.1 & 4 & 0.784 & 4.5 \\
Tavajohi-Kermani et al. & 2002 & USA & OP & 1016 & 6.0 & 3.0 & 0.5 & 8.8 \\
Silva Meza R & 2003 & Mexico & OP & 668 & - & - & - & 2.7 \\
Fekonja A & 2005 & Slovenia & OP & 212 & 7.1 & 4.2 & 0.591 & 11.3 \\
Endo et al. & 2006 & Japan & OP & 3358 & 9.3 & 8.5 & 0.914 & 7.5 \\
Sisman et al. & 2007 & Turkey & OP & 2413 & 7.5 & 8.1 & 1.08 & 6.5 \\
Altug-Atac AT, Erdem D & 2007 & Turkey & OP & 3043 & 3.1 & 2.1 & 0.677 & 2.6 \\
Goya et al. & 2008 & Japan & PeDP & 2072 & 10.8 & 8.7 & 0.806 & 9.4 \\
Chung et al. & 2008 & Korea & OP & 1622 & 11.3 & 11.1 & 0.982 & 11.2 \\
Celikoglu et al. & 2010 & Turkey & OP & 3341 & 4.8 & 3.7 & 0.770 & 4.3 \\
Gomes et al. & 2010 & Brazil & OP & 1049 & 7.4 & 5.1 & 0.689 & 6.3 \\
Tallón-Walton et al. & 2010 & Spain & PuDP & 1518 & 6.9 & 7.7 & 1.116 & 7.25 \\
Behr et al. & 2011 & Germany & OP & 1353 & 12.5 & 9.9 & 0.694 & $11.3^{*}$
\end{tabular}

OP: orthodontic patients; SC: schoolchildren; PuDP: public dental patients; PeDP: pediatric dental patients; M:F: Male-to-female ratio * $12.63 \%$ missing prevalence, including oligodontia and cleft palate.

The prevalence of hypodontia varies according to sex, race, geographic and demographic distribution, as well as ethnic affiliation ${ }^{2,10,11}$. Evolutionary changes also have affect on diversity. Some researchers in their studies have come to a conclusion that the rate of hypodontia increases in time ${ }^{2,11,16}$, while others do not confirm that ${ }^{10,12}$.

Tooth agenesis can be represented in both, primary and permanent dentition. In primary dentition it is represented from $0.1 \%-0.9 \%$. In $30-50 \%$ of the cases tooth agenesis in primary dentition is followed with agenesis of their permanent replacements.

Tooth agenesis presents an important clinical and social health problem. The early diagnosis of this anomaly may alert the clinician of possible presence of other associated dental anomaly in the same patient or his family. This is order to prevent the possible consequences. The diagnosis usually is made after radiographic examination. However, germs of the teeth that erupt later are formed later, so their early diagnosis is not possible by radiographic status. Dental examination along with radiographic screening for possible presence of hypodontia in early childhood should be emphasized as part of a public dental health policy. This will help in early diagnosis and will enable timely intervention. Tooth agenesis with its aesthetic and functional influence, its diagnosis, prevention and therapy become an integral part of comprehensive dental health care for the patient.

The aim of this study was to calculate the prevalence of tooth agenesis of the FYROM's population, to see if there is a difference between the 2 largest ethnic communities, Macedonian and Albanian; then, how is tooth agenesis distributed between genders, to confirm the hypothesis that it is more common in females; which teeth are most commonly missing, whether those that are last formed in a given class (incisors, premolars, molars); then, what is the total number of missing teeth in one patient with hypodontia, their distribution between the maxilla and mandible and whether those patients who lack 2 or more teeth have unilateral or bilateral distribution.

\section{Material and Methods}

In this retrospective study, patients' dental history was examined along with their panoramic radiographs. The examination took place at the Clinic of orthodontics at the Faculty of Dentistry in Skopje and included patients from all regions in FYROM in period from 1998 till 2013. Patients with a history of extracted teeth due to trauma, dental decay or periodontal tissue loss were excluded from the study. The study included only respondents who had proper anamnesis and panoramic radiograph in their dental history. The patients were 8-18 years old and grouped by gender and ethnicity. In those patients where tooth agenesis was confirmed, it was recorded which tooth/teeth was/were missing. Tooth agenesis of third molars was excluded from the study.

The collected data was statistically analyzed with programme Statistica 7.0. 


\section{Results}

The total number of participants who met the criteria of this study was $8160.78 .15 \%$ were of Macedonian ethnic community, while remaining $21.85 \%$ of Albanian. Of all participants $44.99 \%$ were males, while $55.01 \%$ females. The mean age of all patients that enrolled this study was 11.29 years (Fig. 1)

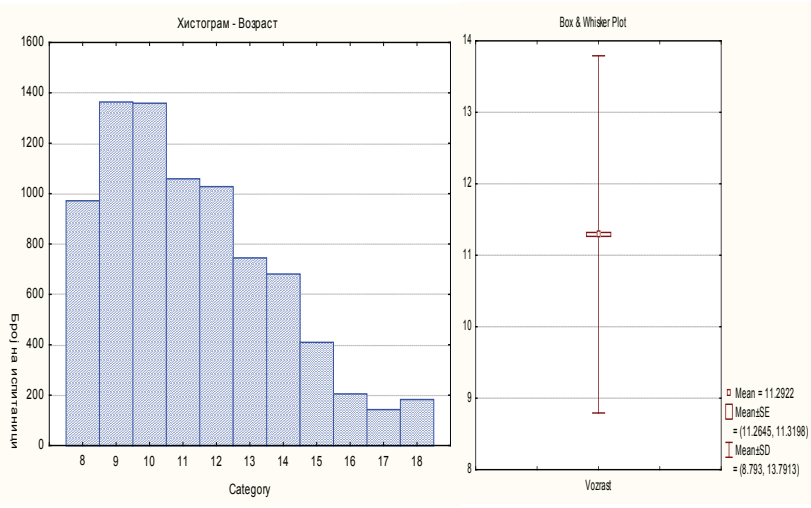

Figure 1. Examinees age distribution and average age

The results showed that prevalence of hypodontia in FYROM is $7.52 \%$. According to Mann-Whitney test, in terms of ethnicity, the difference was not significant for $\mathrm{P}$ value $<0.05(\mathrm{P}=0.31)$. The prevalence of hypodontia among Macedonian community is $7.18 \%$, while it was $8.75 \%$ in Albanian community (Tab. 2). The difference was not significant either in relation to gender, $\mathrm{P}<0.05$ $(\mathrm{P}=0.13)$. Prevalence among male examinees was $6.46 \%$ and $8.40 \%$ in females (Tab. 3). The distribution in terms of jaws involvement was as follows: mandible $43.97 \%$, maxilla $38.11 \%$, while in $17.92 \%$ of the cases there were missing teeth in both jaws. Unilateral distribution was present in $52.12 \%$ and bilateral in $47.88 \%$ of the cases. Those patients that had tooth agenesis of 2 or more teeth in $94.53 \%$ of the cases it was bilateral.

Table 2. Correlation of patients according to ethnicity and presence of hypodontia

$\begin{array}{lccc} & \begin{array}{c}\text { Without } \\ \text { hypodontia }\end{array} & \text { With hypodontia } & \text { Total } \\ \text { Macedonians } & 5918 & 458(7,18 \%) & 6376 \\ \text { Albanians } & 1628 & 156(8,75 \%) & 1784 \\ \text { Total } & 7546 & 614(7,52 \%) & 8160\end{array}$

Table 3. Correlation of patients according to sex and presence of hypodontia

$\begin{array}{lccc} & \begin{array}{c}\text { Without } \\ \text { hypodontia }\end{array} & \begin{array}{c}\text { With } \\ \text { hypodontia }\end{array} & \text { Total } \\ \text { Male } & 3434 & 237(6,46 \%) & 3671 \\ \text { Female } & 4112 & 377(8,40 \%) & 4489 \\ \text { Total } & 7546 & 614(7,52 \%) & 8160\end{array}$

Average number of congenitally missing teeth in patients with hypodontia of FYROM population was 2.04. Out of all examinees, $49.35 \%$ were missing only 1 tooth, while maximal number of missing teeth was 17 , found in 1 person (Fig. 2). The most common missing tooth was lower second premolar. The left one was presented in $35.50 \%$ of the patients with hypodontia, while the right one in $34.53 \%$. This one was followed by upper lateral incisor, right one presented in $27.03 \%$ and left one in $26.55 \%$ of the cases. Least affected were upper first molars with $0.16 \%$ each. In the mandible least affected were canines, left canine in $0.16 \%$ and right canine in $0.33 \%$ of the patients with hypodontia (Fig. 3).

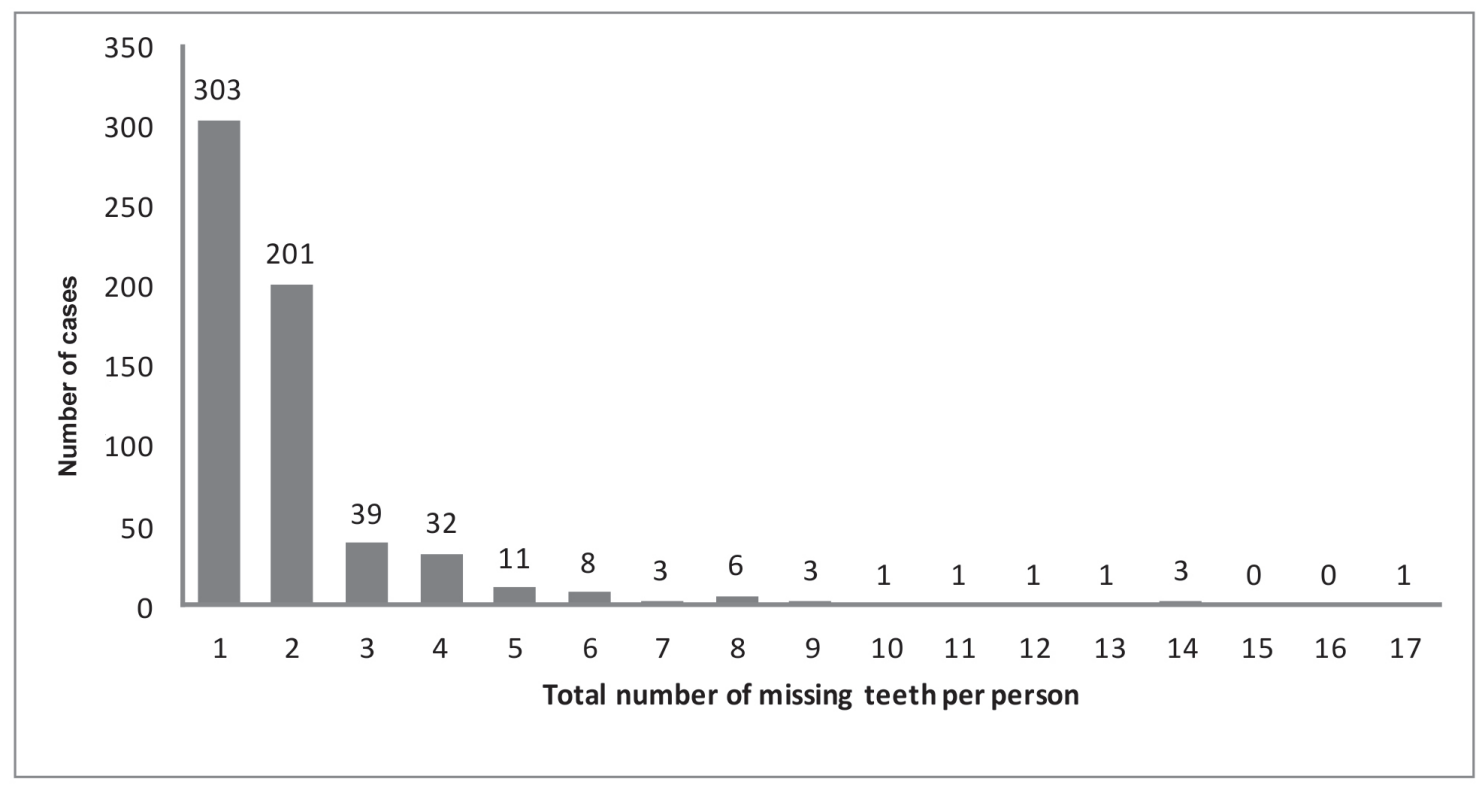

Figure 2. Total number of congenitally missing teeth per person 


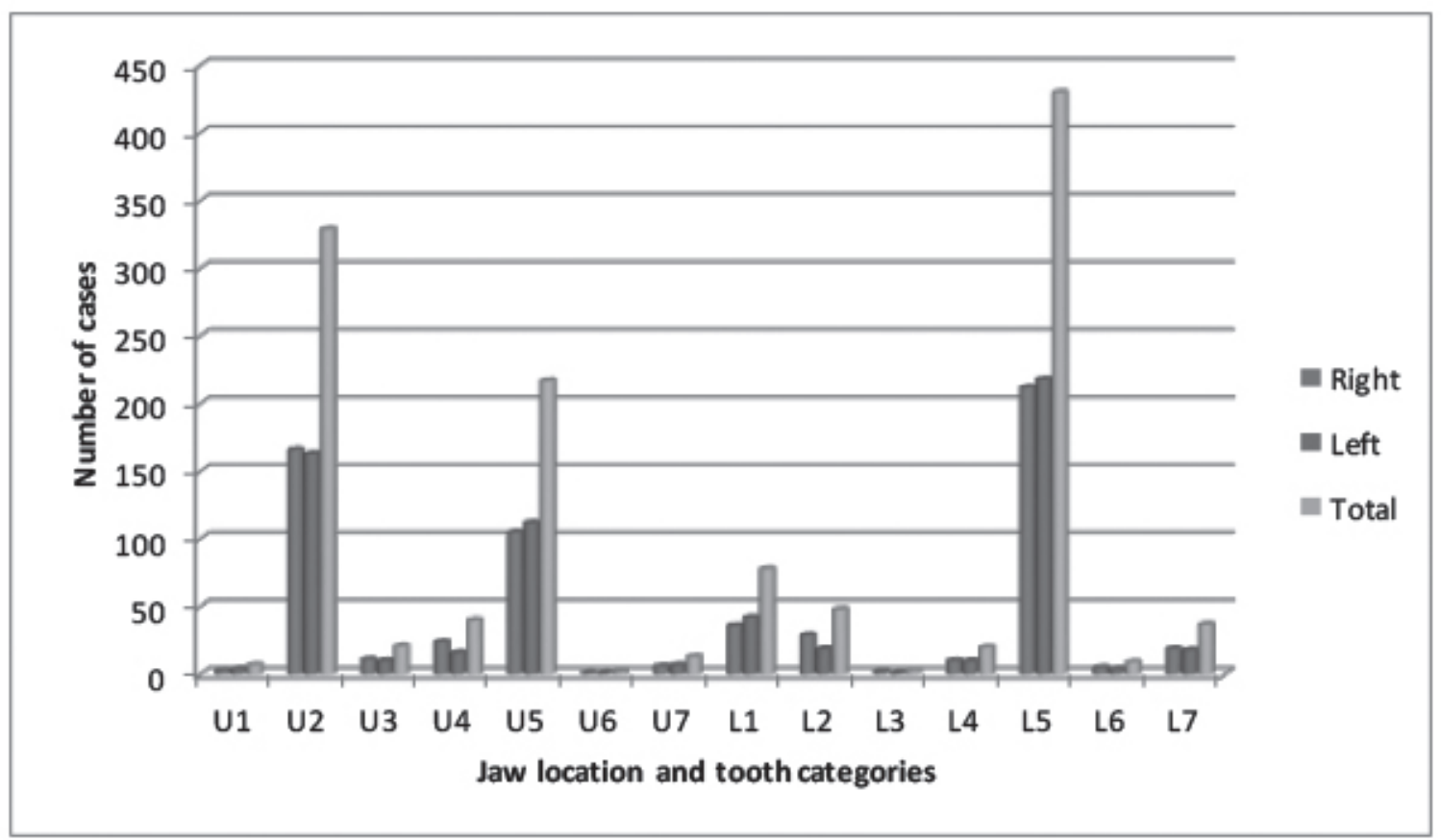

Figure 3. Frequency distribution of missing teeth in Macedonian population

\section{Discussion}

Hypodontia is one of the most common dentofacial anomalies. Due to its frequency, complexity in its approach and method of treatment, it is a subject of many scientific papers. Epidemiological studies get us closer to its distribution among different populations. The purpose of these examinations is to calculate its prevalence in the general population, the ratio between the sexes, which teeth are most affected, what is their distribution by quadrants, which jaw is more affected, whether it is unilateral or bilateral and what is the total number of missing teeth in an individual with hypodontia. Also, through these studies, we can see a distribution among different ethnic groups, racial allocation and demographic distribution.

Through meta-analysis in patients with hypodontia it is proven that the presence of this anomaly in Caucasians is higher than in Negro race. It is also higher in Europe and Australia than in North America. Tooth agenesis is greater among women than among men. In Portugal in 2012 the prevalence of hypodontia was $6.1 \%$. It was more prevalent in women than in men, although this difference was not statistically significant ${ }^{17}$. In Iran, in accordance with a study made in 2012, the prevalence of hypodontia was calculated at $5.21 \%$. The difference in terms of sex was not significant, although women predominated with $5.86 \%$ rather than men with $4.25 \%$. Average number of missing teeth in patients with hypodontia was 1.69 . It is interesting that although the percentage of patients with hypodontia is bigger in females, males are those who have more missing teeth per person (2.32 in men and 1.40 in women). This tells us that hypodontia is more severe in men. Most common missing tooth was maxillary lateral incisor with $37.2 \%$, followed by mandibular second premolar $22.1 \%$ and mandibular central incisor $10.7 \%$. The difference between missing teeth in maxilla and mandible was significant. Maxilla predominated with $5.3 \%$, while mandible was affected in $3.5 \%$ of the cases (Fig. 4$)^{18}$.

Study made in Venezuela in 2012 showed similar results. Prevalence of hypodontia was $4 \%$. Half of the examinees had 1 missing tooth, and other half more than 1 tooth. Women were more affected compared to males at ratio 1.5:1. Most common missing tooth was upper lateral incisor with $40 \%$ of patients with tooth agenesis. Maxilla was more affected with $55 \%$ than the mandible with $45 \%{ }^{19}$. In epidemiological study conducted in Turkey in 2011, the prevalence of hypodontia was $6.77 \%$, with no significant difference between the sexes $(7.63 \%$ in females and $5.44 \%$ in males). The most frequent missing tooth was maxillary lateral incisor, followed by mandibular second premolar $^{20}$. In Egypt population the prevalence of hypodontia amounted to $2.4 \%$ which is the lowest compared to the other countries ${ }^{21}$.

In Japan the presence of hypodontia was $8.5 \%$. Women were more dominant with representation of $9.3 \%$, while men were represented with $7.5 \%$. However this difference was not significant. The most common missing tooth was mandibular second premolar, followed by maxillary and mandibular lateral incisor. In patients with hypodontia average tooth missing per person was $2.4^{22}$. 
The highest prevalence of congenitally missing teeth was recorded in the Republic of Korea in 2011 with $11.3 \%$ - the most frequent missing tooth was mandibular second premolar with $44.2 \%$ prior to mandibular lateral incisor with $36.6 \%$. In women the prevalence was
$12.4 \%$ and in men $9.5 \%$. Half of the examinees were missing only 1 tooth, $36 \%$ were missing 2 , while the rest were missing more than 2 teeth. Figure 5 presents the proportional presence for every missing tooth separately ${ }^{23}$.

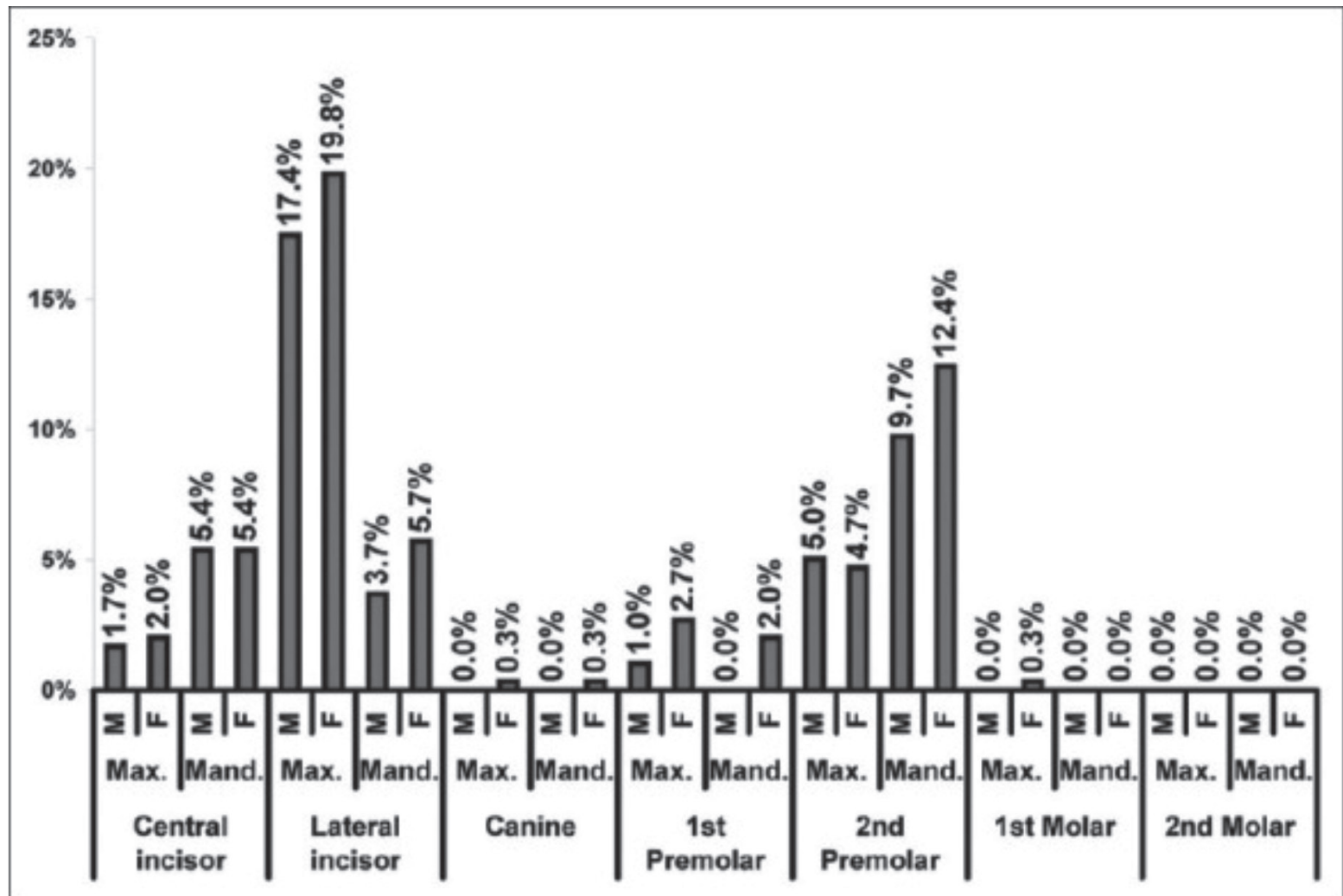

Figure 4. Frequency distribution of missing teeth in Iranian population in 2012

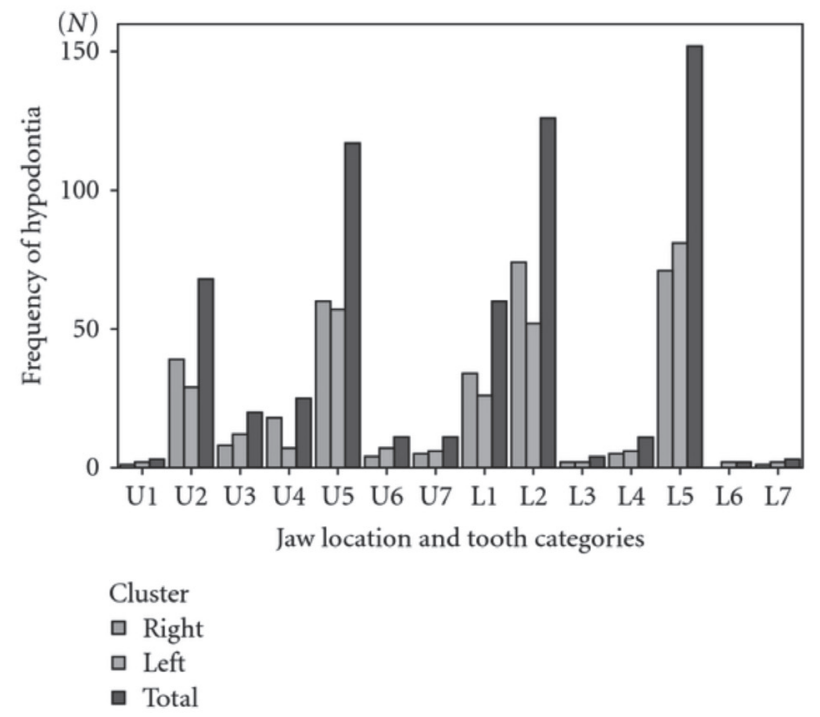

Figure 5. Frequency distribution of missing teeth in Republic of Korea

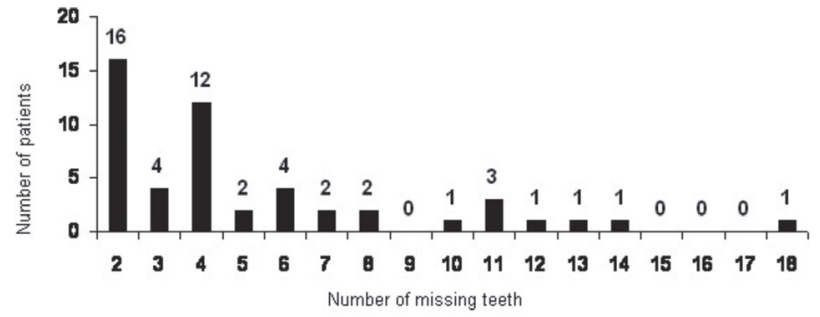

Figure 6. Total number of missing teeth in a study of 50 examinees that are missing more than one tooth

Figure 6 shows the total number of missing teeth in an individual in a study performed in 50 patients with hypodontia of more than one tooth ${ }^{10}$.

From all mentioned above we can conclude that the prevalence of hypodontia of FYROM population does not deviate from the results of epidemiological studies done in other countries. With prevalence of $7.52 \%$ FYROM 
is situated in the middle. Although the difference is not significant, women are more affected, which confirms the hypothesis that this anomaly is more dominant in females than in males. Though there is insignificant difference between 2 biggest ethnic communities in FYROM.

The results show us that in most of the cases the missing teeth were those teeth which develop last in the sequence of a given group (second premolar, lateral incisor). Lower incisors were the exception from this rule because tooth agenesis was present more in central than in lateral. Bilateral distribution in maxilla was mostly due to bilateral agenesis of the left and right lateral incisors. However, in the mandible it is due to tooth agenesis of left and right second premolar.

Mattheeuws et $\mathrm{al}^{24}$ through meta-analysis, where data was evaluated chronologically, came to an interesting fact where they concluded that the degree of prevalence of patients with hypodontia has ascending trend in recent years and is diagnosed more often in everyday practice ${ }^{1}$.

\section{References}

1. Altug-Atac AT, Erdem D. Prevalence and distribution of dental anomalies in orthodontic patients. Am J Orthod Dentofacial Orthop. 2007; 131:510-514.

2. De Coster PJ, Marks LA, Martens LC, Huysseune A. Dental agenesis: Genetic and clinical perspectives. J Oral Pathol Med. 2009; 38:1-17.

3. Goya HA, Tanaka S, Maeda T, Akimoto Y. An orthopantomographic study of hypodontia in permanent teeth of Japanese pediatric patients. J Oral Sci.

4. Nunn JH, Carter NE, Gillgrass TJ, Hobson RS, Jepson NJ, Meechan JG, et al. The interdisciplinary management of hypodontia: Background and role of paediatric dentistry. $\mathrm{Br}$ Dent J. 2003; 194:245-251.

5. Pemberton TJ, Das P, Patel PI. Hypodontia: Genetics and future perspectives. Braz J Oral Sci. 2005;4:695-709

6. Nik-Hussein NN. Hypodontia in the permanent dentition: A study of its prevalence in Malaysian children. Aust Orthod J. 1989; 11:93-95.

7. Gomes RR, da Fonseca JAC, Paula LM, Faber J, Acevedo $A C$. Prevalence of hypodontia in orthodontic patients in Brasilia, Brazil. Eur J Orthod. 2010; 32:302-306.

8. Tallón-Walton $V$, Nieminen $P$, Arte $S$, Carvalho-Lobato P, Ustrell-Torrent JM, Manzanares-Céspedes MC. An epidemiological study of dental agenesis in a primary health area in Spain: Estimated prevalence and associated factors. Med Oral Patol Oral Cir Bucal. 2010; 15:e569-74.

9. Wu CC-L, Wong $R W-K$, Hägg $U$. A review of hypodontia: the possible etiologies and orthodontic, surgical and restorative treatment options - conventional and futuristic. Hong Kong Dent J. 2007; 4:113-121.
10. Amelia Krezci, Peter Proff, Claudia Reicheneder, Andreas Faltermeier. Effects of hypodontia on craniofacial and mandibular growth pattern. Head and face medicine, Dec 2011

11. Polder BJ, Van't Hof MA, Van der Linden FP, KuijpersJagtman AM. A meta-analysis of the prevalence of dental agenesis of permanent teeth. Community Dent Oral Epidemiol. 2004; 32:217-26.

12. Sisman Y, Uysal T, Gelgor IE. Hypodontia.Does the Prevalence and Distribution Pattern Differ in Orthodontic Patients? Eur J Dent. 2007; 1:167-73.

13. Chung CJ, Han JH, Kim KH. The pattern and prevalence of hypodontia in Koreans. Oral Dis. 2008; 14:620-625.

14. Kokich VG, Kokich VO. Congenitally missing mandibular second premolars: Clinical options. Am $J$ Orthod Dentofacial Orthop. 2006; 130:437-444.

15. Larmour CJ, Mossey PA, Thind BS, Forgie AH, Stirrups $D R$. "Hypodontia - a retrospective review of prevalence and etiology”. Quintessence Int, 2005; 36(4):263-270.

16. Flores-Mir $C$. Increased hypodontia through the twentieth century. Evid Based Dent. 2006; 7:15.

17. González-Allo A, Campoy MD, Moreira J, Ustrell J, Pinho $T$. Tooth agenesis in a Portuguese population. Int Orthod, 2012; 10(2):198-210.

18. Fariborz Amini, Vahid Rakhshan, and Pardis Babaei. Prevalence and pattern of hypodontia in the permanent dentition of 3374 Iranian orthodontic patients. Dent Res $J$ (Isfahan), 2012; 9(3):245-250.

19. Medina AC. Radiographic study of prevalence and distribution of hypodontia in a pediatric orthodontic population in Venezuela. Pediatr Dent, 2012; 34(2):e113-16.

20. Topkara A, Sari Z. Prevalence and distribution of hypodontia in a Turkish orthodontic patient population: results from a large academic cohort. Eur J Paediatr Dent, 2011; 12(2):123-127.

21. Montasser MA, Taha M. Prevalence and distribution of dental anomalies in orthodontic patients. Orthodontics (Chic.), 2012; 13(1):52-59.

22. Endo T, Ozoe R, Kubota M, Akiyama M, Shimooka S. A survey of hypodontia in Japanese orthodontic patients. Am J Orthod Dentofacial Orthop, 2006; 129(1):29-35.

23. Young Ho Kim. Investigation of Hypodontia as Clinically Related Dental Anomaly: Prevalence and Characteristics. ISRN Dent, 2011.

24. Mattheeuws N, Dermaut L, Martens G. Has hypodontia increased in Caucasians during the $20^{\text {th }}$ century? A metaanalysis. European Journal of Orthodontics, 2004 June

Correspondence and request for offprints to:

Darko Pop Acev

Ul. 8 Mart 8a

1000 Skopje

FYR Macedonia

E-mail: darko-pop-acev@hotmail.com 